TOPOLOGICAL ALGEBRAS, THEIR APPLICATIONS,

AND RELATED TOPICS

BANACH CENTER PUBLICATIONS, VOLUME 67

INSTITUTE OF MATHEMATICS

POLISH ACADEMY OF SCIENCES

WARSZAWA 2005

\title{
THE CENTER OF TOPOLOGICALLY PRIMITIVE GALBED ALGEBRAS
}

\author{
MART ABEL \\ Institute of Pure Mathematics, University of Tartu \\ 2 Liivi St., Room 615, 50409 Tartu, Estonia \\ E-mail:mabel@ut.ee
}

Abstract. It is shown that every unital $\sigma$-complete topologically primitive strongly galbed Hausdorff algebra in which all elements are bounded is central.

\section{Introduction}

1.1. Let $\mathbb{C}$ be the field of complex numbers, $\mathbb{N}=\{0,1,2, \ldots\}$ the set of natural numbers, $\mathbb{Z}^{+}=\{1,2, \ldots\}$ the set of positive integers and $l^{0}$ the set of all $\mathbb{C}$-valued sequences $\left(\alpha_{n}\right)$ where $\alpha_{m} \neq 0$ for only a finite number of elements $\alpha_{m}$. For every $k>0$ let $l^{k}$ be the set of all $\mathbb{C}$-valued sequences $\left(\alpha_{n}\right)$ for which the series

converges, $l=l^{1} \backslash l^{0}$, and

$$
\sum_{v=0}^{\infty}\left|\alpha_{v}\right|^{k}
$$

$$
l^{(0,1]}=\bigcap_{k \in(0,1]} l^{k} .
$$

Let $A$ be an associative topological algebra over $\mathbb{C}$ with separately continuous multiplication (for short, a topological algebra).

Definition 1 . We will say that a topological algebra $A$ is a galbed algebra if there exists a sequence $\left(\alpha_{n}\right) \in l$ such that for each neighbourhood $O$ of zero in $A$ there is another neighbourhood $U$ of zero in $A$ such that

for each $n \in \mathbb{N}$.

$$
\left\{\sum_{k=0}^{n} \alpha_{k} a_{k}: a_{0}, \ldots, a_{n} \in U\right\} \subset O
$$

2000 Mathematics Subject Classification: Primary 46H05; Secondary 46H20.

Key words and phrases: galbed algebras, center of an algebra, primitive algebras, topologically primitive algebras, exponentially galbed algebras.

Research is partly supported by the Estonian Science Foundation grant 4514.

The paper is in final form and no version of it will be published elsewhere. 
Furthermore, if there exists a sequence $\left(\alpha_{n}\right) \in l$ with $\alpha_{0} \neq 0$ and

$$
\alpha=\inf _{n>0}\left|\alpha_{n}\right|^{\frac{1}{n}}>0
$$

such that the previous condition is true, then we say that $A$ is a strongly galbed algebra. We call $\alpha$ the "module of galbness" of $A$.

In case we have already specified the sequence $\left(\alpha_{n}\right) \in l$, then we talk about $\left(\alpha_{n}\right)$-galbed algebra and strongly $\left(\alpha_{n}\right)$-galbed algebra.

For a linear topological space $X$, the notions of $\left(\alpha_{n}\right)$-galbed space and galbed space are defined similarly (see [8]). It is clear, that every $\left(\alpha_{n}\right)$-galbed algebra is an $\left(\alpha_{n}\right)$-galbed space and every galbed algebra is a galbed space.

Recall that a topological algebra $A$ is locally pseudoconvex if it has a base $\left\{U_{\lambda}: \lambda \in \Lambda\right\}$ of neighbourhoods of zero consisting of balanced and pseudoconvex sets (that is, of sets $U$ for which $\mu U \subset U$, whenever $|\mu| \leq 1$, and $U+U \subset \rho U$ for a $\rho \geq 2$ ). In particular, when every $U_{\lambda}$ in $\left\{U_{\lambda}: \lambda \in \Lambda\right\}$ is idempotent (that is, $U_{\lambda} U_{\lambda} \subset U_{\lambda}$ ), then $A$ is called a locally m-pseudoconvex algebra, and when every $U_{\lambda}$ in $\left\{U_{\lambda}: \lambda \in \Lambda\right\}$ is $A$-pseudoconvex (that is, for any $a \in A$ there is a $\mu>0$ such that $a U_{\lambda}, U_{\lambda} a \subset \mu U_{\lambda}$ ), then $A$ is called a locally $A$-pseudoconvex algebra. It is well known (see [20], p. 4, or [9], p. 189) that the locally pseudoconvex topology on $A$ can be given by a family $\left\{p_{\lambda}: \lambda \in \Lambda\right\}$ of $k_{\lambda}$-homogeneous seminorms, where $k_{\lambda} \in(0,1]$ for each $\lambda \in \Lambda$. The topology of a locally $m$-pseudoconvex ( $A$-pseudoconvex) algebra $A$ can be given by a family $\left\{p_{\lambda}: \lambda \in \Lambda\right\}$ of $k_{\lambda}$-homogeneous submultiplicative ${ }^{1}$ (respectively, $A$-multiplicative ${ }^{2}$ ) seminorms, where $k_{\lambda} \in(0,1]$ for each $\lambda \in \Lambda$. In particular, when $k_{\lambda}=1$ for each $\lambda \in \Lambda$, then $A$ is a locally convex (respectively, locally $m$-convex and locally $A$-convex) algebra, and when the topology of $A$ has been defined by only one $k$-homogeneous seminorm with $k \in(0,1]$, then $A$ is a locally bounded algebra. Moreover, a complete locally bounded Hausdorff algebra $A$ is a $k$-Banach algebra for some $k \in(0,1]$, a complete metrizable algebra $A$ is a Fréchet algebra, a sequentially complete algebra is a $\sigma$-complete algebra and a unital topological algebra $A$ in which the set of all invertible elements is open (the center $Z(A)$ of $A$ is topologically isomorphic to $\mathbb{C}$ ) is a $Q$-algebra (respectively, a central algebra). An algebra $A$ is an exponentially galbed algebra (see, for example, [1], [2], [3], [4], [5], [6], [18] and [19]) if for every neighbourhood $O$ of $A$ there is another neighbourhood $U$ of zero such that

$$
\left\{\sum_{k=0}^{n} \frac{a_{k}}{2^{k}}: a_{0}, \ldots, a_{n} \in U\right\} \subset O
$$

for each $n \in \mathbb{N}$. It is easy to see that every locally pseudoconvex algebra is an exponentially galbed algebra.

Notice that every $\left(2^{-n}\right)$-galbed algebra is an exponentially galbed algebra, every locally pseudoconvex algebra is an $\left(\alpha_{n}\right)$-galbed algebra if $\left(\alpha_{n}\right) \in l^{(0,1]}$, and every locally $k$-convex algebra is an $\left(\alpha_{n}\right)$-galbed algebra if $\left(\alpha_{n}\right) \in l^{k}$. Moreover, every exponentially galbed algebra is an $\left(\alpha_{n}\right)$-galbed algebra if $\left|\alpha_{n}\right| \leq 2^{-n}$ for each $n \in \mathbb{N}$, and every $\left(\alpha_{n}\right)$-galbed

\footnotetext{
${ }^{1}$ That is, $p_{\lambda}(a b) \leq p_{\lambda}(a) p_{\lambda}(b)$ for each $a, b \in A$ and $\lambda \in \Lambda$.

${ }^{2}$ That is, for each $a \in A$ and each $\lambda \in \Lambda$ there are numbers $N(a, \lambda)>0$ and $M(a, \lambda)>0$ such that $p_{\lambda}(a b) \leq N(a, \lambda) p_{\lambda}(b)$ and $p_{\lambda}(b a) \leq M(a, \lambda) p_{\lambda}(b)$ for each $b \in A$.
} 
algebra is an exponentially galbed algebra if $\left|\alpha_{n}\right| \geq 2^{-n}$ for each $n \in \mathbb{N}$. Hence, the class of galbed algebras is much larger than the class of exponentially galbed algebras.

A topological algebra $A$ is a topologically primitive algebra (see [7]) if $\{a \in A$ : $a A \subset M\}=\left\{\theta_{A}\right\}\left(\{a \in A: A a \subset M\}=\left\{\theta_{A}\right\}\right)$ for a closed maximal regular (or modular) left (respectively, right) ideal $M$ of $A$ (here $\theta_{A}$ denotes the zero element of $A$ ). Recall that a ring (in particular, algebra) $R$ is primitive if it has a maximal regular left (respectively, right) ideal $M$ such that $\{a \in R: a R \subset M\}=\left\{\theta_{R}\right\}$ (respectively, $\left.\{a \in R: R a \subset M\}=\left\{\theta_{R}\right\}\right)$. An element $a$ in a topological algebra $A$ is bounded if there exists a number $\lambda_{a} \in \mathbb{C} \backslash\{0\}$ such that the set

$$
\left\{\left(\frac{a}{\lambda_{a}}\right)^{n}: n \in \mathbb{Z}^{+}\right\}
$$

( $n \in \mathbb{N}$, if $A$ is unital) is bounded in $A$. If all elements in $A$ are bounded, then $A$ is a topological algebra with bounded elements. An element $a \in A$ is nilpotent if $a^{m}=\theta_{A}$ for some $m \in \mathbb{N}$. If all elements in $A$ are nilpotent, then $A$ is called a nil algebra.

1.2. It is well known that the center of a primitive ring is an integral domain ${ }^{3}$ (see [12], Lemma 2.1.3, p. 45) and any commutative integral domain can be the center of a primitive ring $^{4}$ (see [13], Chapter II.6, Example 3, p. 36). Recall that every field is a commutative integral domain, but a commutative integral domain is not necessarily a field. In particular (see [7]), when $R$ is a unital primitive locally $A$-pseudoconvex Hausdorff algebra or a unital primitive locally pseudoconvex Fréchet $Q$-algebra, then $R$ is central (for Banach algebras a similar result is given in [15], Corollary 2.4.5, see also [10], p. 127; [14], Theorem 4.2.11, and [11], Theorem 2.6.26 (ii); for $k$-Banach algebras in [9], Corollary 9.3.7; for locally $m$-convex $Q$-algebras in [16], Corollary 2 , and for locally $A$ convex algebras in which all maximal ideals are closed in [17], Theorem 3). In [4] it was shown that a unital $\sigma$-complete topologically primitive exponentially galbed Hausdorff algebra with bounded elements is central.

In the present paper we will show that a similar result will be true for any unital $\sigma$-complete topologically primitive strongly galbed Hausdorff algebra in which all elements are bounded.

2. Auxiliary results. Let $M$ be a closed linear subspace of a linear topological space $X$. By $X / M$ we denote the quotient space of $X$ with respect to $M$. To describe the center of primitive galbed algebras we need the following results.

Proposition 2.1. Let $X$ be a (strongly) galbed space. If $M$ is a closed linear subspace of $X$, then $X / M$ is a (strongly) galbed (Hausdorff) space.

Proof. Let $\tau$ be the topology on $X$ such that $(X, \tau)$ is an $\left(\alpha_{n}\right)$-galbed space. Let $M$ be a closed linear subspace of $X$ and $\tau_{M}$ the quotient topology on $X / M$, defined by $\tau$. Let $\pi: X \rightarrow X / M$ be the canonical homomorphism and $O$ a neighbourhood of zero in

\footnotetext{
${ }^{3} \mathrm{~A}$ ring $R$ is an integral domain, if from $a, b \in R$ and $a b=\theta_{R}$ follows that $a=\theta_{R}$ or $b=\theta_{R}$.

${ }^{4}$ The author would like to express his gratitude to Professor Laszlo Marki for informing him about this result.
} 
$\left(X / M, \tau_{M}\right)$. Then $\pi$ is continuous and open. Therefore, $O^{\prime}=\pi^{-1}(O)$ is a neighbourhood of zero in $(X, \tau)$ and there exists a neighbourhood $V$ of zero in $X$ such that

$$
\left\{\sum_{k=0}^{n} \alpha_{k} v_{k}: v_{0} \ldots, v_{n} \in V\right\} \subset O^{\prime}
$$

for each $n \in \mathbb{N}$. Now, $U=\pi(V)$ is a neighbourhood of zero in $\left(X / M, \tau_{M}\right)$ such that

$$
\left\{\sum_{k=0}^{n} \alpha_{k} u_{k}: u_{0}, \ldots, u_{n} \in U\right\} \subset O
$$

for each $n \in \mathbb{N}$. Thus, $\left(X / M, \tau_{M}\right)$ is an $\left(\alpha_{n}\right)$-galbed (Hausdorff) space.

Proposition 2.2. Let $A$ be a unital strongly galbed Hausdorff algebra with bounded elements, which is also $\sigma$-complete or a nil algebra. Moreover, let $\lambda_{0} \in \mathbb{C}$ and $a_{0} \in A$. Then there exists a neighbourhood $O\left(\lambda_{0}\right)$ of $\lambda_{0}$ such that

$$
\sum_{k=0}^{\infty}\left(\lambda-\lambda_{0}\right)^{k} a_{0}^{k}
$$

converges in $A$ and

$$
\left(e_{A}+\left(\lambda_{0}-\lambda\right) a_{0}\right)^{-1}=\sum_{k=0}^{\infty}\left(\lambda-\lambda_{0}\right)^{k} a_{0}^{k}
$$

for each $\lambda \in O\left(\lambda_{0}\right)$.

Proof. Let $A$ be an $\left(\alpha_{n}\right)$-galbed Hausdorff algebra with bounded elements, $\alpha>0$ and $O$ an arbitrary neighbourhood of zero in $A$. Then there is a closed and balanced neighbourhood $O^{\prime}$ of zero in $A$ and a closed neighbourhood $O^{\prime \prime}$ of zero in $\mathbb{C}$ such that $O^{\prime \prime} O^{\prime} \subset O$. Now $O^{\prime}$ yields a balanced neighbourhood $V$ of zero in $A$ such that

$$
\left\{\sum_{k=0}^{n} \alpha_{k} v_{k}: v_{0}, \ldots, v_{n} \in V\right\} \subset O^{\prime}
$$

for each $n \in \mathbb{N}$. Since every element in $A$ is bounded, there is a number $\mu_{0}=\mu_{a_{0}} \in \mathbb{C} \backslash\{0\}$ such that

$$
\left\{\left(\frac{a_{0}}{\mu_{0}}\right)^{n}: n \in \mathbb{N}\right\}
$$

is bounded in $A$. Therefore, there exists a number $\rho_{0}>0$ such that

$$
\left(\frac{a_{0}}{\mu_{0}}\right)^{n} \in \rho_{0} V \cap \rho_{0} \alpha_{0} V
$$

for each $n \in \mathbb{N}$.

Let now $a_{0} \in A$ and $\lambda_{0} \in \mathbb{C}$ be fixed,

$$
S_{n}(\lambda)=\sum_{k=0}^{n}\left(\lambda-\lambda_{0}\right)^{k} a_{0}^{k}
$$

for each $n \in \mathbb{N}$ and $\lambda \in \mathbb{C}$,

$$
U_{\mathbb{C}}=\left\{\lambda \in \mathbb{C}:|\lambda|<\frac{\alpha}{\left|\mu_{0}\right|}\right\}
$$


and $O\left(\lambda_{0}\right)=\lambda_{0}+U_{\mathbb{C}}$. Then

$$
S_{m}(\lambda)-S_{n}(\lambda)=\sum_{k=n+1}^{m}\left(\lambda-\lambda_{0}\right)^{k} a_{0}^{k}=\sum_{k=0}^{m-n-1}\left(\lambda-\lambda_{0}\right)^{n+k+1} a_{0}^{n+k+1}
$$

for each $n, m \in \mathbb{N}$, whenever $m>n$ and $\lambda \in \mathbb{C}$. If we take

$$
v_{n, k}(\lambda)=\left(\lambda-\lambda_{0}\right)^{k} \frac{a_{0}^{n+k+1}}{\rho_{0} \alpha_{k} \mu_{0}^{n+1}}
$$

for each $n, k \in \mathbb{N}$ and $\lambda \in \mathbb{C}$, then

$$
S_{m}(\lambda)-S_{n}(\lambda)=\left(\lambda-\lambda_{0}\right)^{n+1} \mu_{0}^{n+1} \rho_{0} \sum_{k=0}^{m-n-1} \alpha_{k} v_{n, k}(\lambda)
$$

for each $n, m \in \mathbb{N}$, whenever $m>n$ and $\lambda \in \mathbb{C}$. Now,

$$
v_{n, 0}(\lambda)=\frac{1}{\rho_{0} \alpha_{0}}\left(\frac{a_{0}}{\mu_{0}}\right)^{n+1} \in V
$$

and

$$
v_{n, k}(\lambda)=\frac{1}{\rho_{0}}\left(\frac{\left(\lambda-\lambda_{0}\right) \mu_{0}}{\alpha}\right)^{k} \frac{\alpha^{k}}{\alpha_{k}}\left(\frac{a_{0}}{\mu_{0}}\right)^{n+k+1} \in \frac{1}{\rho_{0}}\left(\frac{\left(\lambda-\lambda_{0}\right) \mu_{0}}{\alpha}\right)^{k} \frac{\alpha^{k}}{\alpha_{k}} \rho_{0} V \subset V
$$

for each $n \in \mathbb{N}, k \in \mathbb{Z}^{+}$and $\lambda \in O\left(\lambda_{0}\right)$, because $\left|\left(\lambda-\lambda_{0}\right) \mu_{0}\right|<\alpha$ and $\alpha^{k} \leq \alpha_{k}$ for each $k \in \mathbb{Z}^{+}$. Hence,

$$
S_{m}(\lambda)-S_{n}(\lambda) \in\left(\left(\lambda-\lambda_{0}\right) \mu_{0}\right)^{n+1} \rho_{0} O^{\prime},
$$

whenever $m>n$ and $\lambda \in O\left(\lambda_{0}\right)$. Since again $\left|\left(\lambda-\lambda_{0}\right) \mu_{0}\right|<\alpha<1$, there exists a number $n_{0} \in \mathbb{N}$ such that

$$
\left(\left(\lambda-\lambda_{0}\right) \mu_{0}\right)^{n+1} \in \frac{1}{\rho_{0}} O^{\prime \prime}
$$

for each $n>n_{0}$. Taking this into account,

$$
S_{m}(\lambda)-S_{n}(\lambda) \in \frac{1}{\rho_{0}} O^{\prime \prime} \rho_{0} O^{\prime} \subset O^{\prime \prime} O^{\prime} \subset O,
$$

whenever $m>n>n_{0}$ and $\lambda \in O\left(\lambda_{0}\right)$, since $O^{\prime}$ is balanced. This means that $\left(S_{n}(\lambda)\right)$ is a Cauchy sequence in $A$ for each fixed $\lambda \in O\left(\lambda_{0}\right)$.

In the case when $A$ is $\sigma$-complete, the sequence $\left(S_{n}(\lambda)\right)$ converges in $A$. But if $A$ is not $\sigma$-complete, let $A$ be a nil algebra. Then $a_{0}^{m+1}=\theta_{A}$ for some $m \in \mathbb{N}$. Hence,

$$
S_{n}(\lambda)=\sum_{k=0}^{m}\left(\lambda-\lambda_{0}\right)^{k} a_{0}^{k}
$$

for each $\lambda \in \mathbb{C}$, whenever $n \geq m$. Consequently, $\left(S_{n}(\lambda)\right)$ converges in $A$ for each $\lambda \in O\left(\lambda_{0}\right)$ in both cases.

Because

$$
\left(e_{A}+\left(\lambda_{0}-\lambda\right) a_{0}\right) \sum_{k=0}^{\infty}\left(\lambda-\lambda_{0}\right)^{k} a_{0}^{k}=\sum_{k=0}^{\infty}\left(\lambda-\lambda_{0}\right)^{k} a_{0}^{k}\left(e_{A}+\left(\lambda_{0}-\lambda\right) a_{0}\right)=e_{A},
$$


we have

$$
\left(e_{A}+\left(\lambda_{0}-\lambda\right) a_{0}\right)^{-1}=\sum_{k=0}^{\infty}\left(\lambda-\lambda_{0}\right)^{k} a_{0}^{k}
$$

for each $\lambda \in O\left(\lambda_{0}\right)$.

Corollary 2.3. Let $A$ be a unital strongly galbed Hausdorff algebra with bounded elements. If $A$ is a $\sigma$-complete or a nil algebra, then for each $a_{0} \in A$ there exists a number $R>0$ such that

$$
\sum_{k=0}^{\infty} \frac{a_{0}^{k}}{\mu^{k+1}}
$$

converges in $A$, whenever $|\mu|>R$.

Proof. If we take $\lambda_{0}=0$ in the previous proposition, then we get that

$$
\sum_{k=0}^{\infty} \lambda^{k} a_{0}^{k}
$$

converges in $A$, whenever $|\lambda|<\delta$ for some $\delta>0$. If now $|\mu|>R=\delta^{-1}$, then $\left|\mu^{-1}\right|<\delta$ which means that

$$
\sum_{k=0}^{\infty} \frac{a_{0}^{k}}{\mu^{k}}
$$

converges in $A$. Hence,

$$
\sum_{k=0}^{\infty} \frac{a_{0}^{k}}{\mu^{k+1}}=\frac{1}{\mu} \sum_{k=0}^{\infty} \frac{a_{0}^{k}}{\mu^{k}}
$$

converges in $A$, whenever $|\mu|>R$.

3. Main result. Now, by Proposition 2.2 and Corollary 2.3, we give a description of the center $Z(A)$ of unital topologically primitive strongly galbed Hausdorff algebras $A$ in which all elements are bounded.

THEOREM 3.1. Let $A$ be a unital $\sigma$-complete topologically primitive strongly galbed Hausdorff algebra with bounded elements. Then $A$ is a central algebra.

Proof. There exists a sequence $\left(\alpha_{n}\right) \in l$ such that $A$ is $\left(\alpha_{n}\right)$-galbed with $\alpha_{0} \neq 0$ and $\alpha=\inf _{n>0}\left|\alpha_{n}\right|^{\frac{1}{n}}>0$. Let $M$ be a closed maximal left ideal ${ }^{5}$ in $A$ such that $\{a \in A$ : $a A \subset M\}=\left\{\theta_{A}\right\}$ (then $M \cap Z(A)=\left\{\theta_{A}\right\}$ ), $\pi_{M}$ a canonical homomorphism from $A$ onto the quotient space $A / M$ of $A$ with respect to $M$ and for each $z \in Z(A) \backslash\left\{\theta_{A}\right\}$ let $K_{z}=\{a \in A: a z \in M\}$. Because $m z=z m \in M$ for each $m \in M$ and $e_{A} z=z \notin M$, we have $M \subset K_{z} \neq A$. Hence, $K_{z}$ is a left ideal in $A$. Since the ideal $M$ is maximal, $M=K_{z}$ for each $z \in Z(A) \backslash\left\{\theta_{A}\right\}$.

We will show that for every $z \in Z(A)$ there is a number $\lambda_{z} \in \mathbb{C}$ such that $z=\lambda_{z} e_{A}$. If $z=\theta_{A}$, then we take $\lambda_{z}=0$. Suppose now that there exists a $z \in Z(A) \backslash\left\{\theta_{A}\right\}$ such that $z(\lambda)=\lambda e_{A}-z \neq \theta_{A}$ for all $\lambda \in \mathbb{C}$. Then $z(\lambda) \in Z(A) \backslash\left\{\theta_{A}\right\}$ means that $z(\lambda) \notin M$ for each $\lambda \in \mathbb{C}, M+A z(\lambda)$ is a left ideal in $A, M \subset M+A z(\lambda)$ and $z(\lambda)=\theta_{A}+e_{A} z(\lambda) \in$

\footnotetext{
${ }^{5}$ If $M$ is a closed maximal right ideal, then the proof is similar.
} 
$(M+A z(\lambda)) \backslash M$ for each $\lambda \in \mathbb{C}$. Since $M$ is a maximal left ideal in $A$, we have $M+A z(\lambda)=$ $A$ for each $\lambda \in \mathbb{C}$. Therefore, for each $\lambda \in \mathbb{C}$, there are $m(\lambda) \in M$ and $a(\lambda) \in A$ such that $e_{A}=m(\lambda)-a(\lambda) z(\lambda)$, because of which $a(\lambda) z(\lambda)+e_{A} \in M$.

Let $a^{\prime}(\lambda) \in A$ be another element such that $a^{\prime}(\lambda) z(\lambda)+e_{A} \in M$. Then from $[a(\lambda)-$ $\left.a^{\prime}(\lambda)\right] z(\lambda)=a(\lambda) z(\lambda)-a^{\prime}(\lambda) z(\lambda) \in M$ it follows that $\left[a(\lambda)-a^{\prime}(\lambda)\right] \in K_{z(\lambda)}=M$. Thus, $\pi_{M}(a(\lambda))=\pi_{M}\left(a^{\prime}(\lambda)\right)$ for each $\lambda \in \mathbb{C}$.

Moreover, let $\lambda_{0} \in \mathbb{C}$ and $d(\lambda)=e_{A}+\left(\lambda_{0}-\lambda\right) a\left(\lambda_{0}\right)$ for each $\lambda \in \mathbb{C}$. Then there is (by Proposition 2.2) a neighbourhood $O\left(\lambda_{0}\right)$ of $\lambda_{0}$ such that

$$
\sum_{k=0}^{\infty}\left(\lambda-\lambda_{0}\right)^{k} a\left(\lambda_{0}\right)^{k}
$$

converges in $A$ and

$$
d(\lambda)^{-1}=\sum_{k=0}^{\infty}\left(\lambda-\lambda_{0}\right)^{k} a\left(\lambda_{0}\right)^{k}
$$

for each $\lambda \in O\left(\lambda_{0}\right)$. Now

$$
\begin{gathered}
a\left(\lambda_{0}\right) d(\lambda)^{-1} z(\lambda)+e_{A}=a\left(\lambda_{0}\right) d(\lambda)^{-1} z(\lambda)-\left[a\left(\lambda_{0}\right) z\left(\lambda_{0}\right)-m\left(\lambda_{0}\right)\right]= \\
-a\left(\lambda_{0}\right) d(\lambda)^{-1}\left[-z(\lambda)+d(\lambda) z\left(\lambda_{0}\right)\right]+m\left(\lambda_{0}\right)= \\
-a\left(\lambda_{0}\right) d(\lambda)^{-1}\left[\left(z-\lambda e_{A}\right)+\left(e_{A}+\left(\lambda_{0}-\lambda\right) a\left(\lambda_{0}\right)\right)\left(\lambda_{0} e_{A}-z\right)\right]+m\left(\lambda_{0}\right)= \\
-a\left(\lambda_{0}\right) d(\lambda)^{-1}\left[\left(\lambda_{0}-\lambda\right)\left(e_{A}+a\left(\lambda_{0}\right) z\left(\lambda_{0}\right)\right)\right]+m\left(\lambda_{0}\right)= \\
-a\left(\lambda_{0}\right) d(\lambda)^{-1}\left(\lambda_{0}-\lambda\right) m\left(\lambda_{0}\right)+m\left(\lambda_{0}\right) \in M .
\end{gathered}
$$

Therefore, $\pi_{M}(a(\lambda))=\pi_{M}\left(a\left(\lambda_{0}\right) d(\lambda)^{-1}\right)$ for each $\lambda \in O\left(\lambda_{0}\right)$.

Let $\Psi(\lambda)=\pi_{M}(a(\lambda))$ for each $\lambda \in \mathbb{C}$. We will show that $\Psi$ is an $(A / M)$-valued analytic function ${ }^{6}$ on $\mathbb{C} \cup\{\infty\}$. For it, let again $\lambda_{0} \in \mathbb{C}$. Then $\Psi(\lambda)=\pi_{M}\left(a\left(\lambda_{0}\right) d(\lambda)^{-1}\right)$ for each $\lambda \in O\left(\lambda_{0}\right)$ and there exists a number $\delta>0$ such that $\lambda_{0}+\lambda \in O\left(\lambda_{0}\right)$, whenever $|\lambda|<\delta$.

Now,

$$
\Psi\left(\lambda_{0}+h\right)=\pi_{M}\left(a\left(\lambda_{0}\right) d\left(\lambda_{0}+h\right)^{-1}\right)=\pi_{M}\left(a\left(\lambda_{0}\right) \sum_{k=0}^{\infty} h^{k} a\left(\lambda_{0}\right)^{k}\right)=\sum_{k=0}^{\infty} h^{k} \pi_{M}\left(a\left(\lambda_{0}\right)^{k+1}\right),
$$

if $|h|<\delta$, where $\pi_{M}\left(a\left(\lambda_{0}\right)^{k+1}\right) \in A / M$ for each $k \in \mathbb{N}$.

By Corollary 2.3 there is a number $R>0$ such that

$$
\sum_{k=0}^{\infty} \frac{z^{k}}{\lambda^{k+1}}
$$

converges in $A$, if $|\lambda|>R$. Easy calculation shows that

$$
z(\lambda) \sum_{k=0}^{\infty} \frac{z^{k}}{\lambda^{k+1}}=\sum_{k=0}^{\infty} \frac{z^{k}}{\lambda^{k+1}} z(\lambda)=e_{A}
$$

${ }^{6}$ That is, if $\lambda_{0} \in \mathbb{C}$, then there are a number $\delta>0$ and a sequence $\left(x_{n}\right)$ of elements of $A / M$ such that $\Psi\left(\lambda_{0}+\lambda\right)=\sum_{k=0}^{\infty} x_{k} \lambda^{k}$, whenever $|\lambda|<\delta$. Otherwise, there are a number $R>0$ and a sequence $\left(y_{n}\right)$ of elements of $A / M$ such that $\Psi(\lambda)=\sum_{k=0}^{\infty} y_{k} / \lambda^{k}$, whenever $|\lambda|>R$. 
Therefore,

$$
z(\lambda)^{-1}=\sum_{k=0}^{\infty} \frac{z^{k}}{\lambda^{k+1}},
$$

whenever $|\lambda|>R$. Since $z(\lambda)^{-1} z(\lambda)-e_{A} \in M$ for each $\lambda$ with $|\lambda|>R$, we have

$$
\Psi(\lambda)=\pi_{M}\left(z(\lambda)^{-1}\right)=\pi_{M}\left(\sum_{k=0}^{\infty} \frac{z^{k}}{\lambda^{k+1}}\right)=\sum_{k=0}^{\infty} \frac{\pi_{M}\left(z^{k}\right)}{\lambda^{k+1}},
$$

if $|\lambda|>R$, where $\pi_{M}\left(z^{k}\right) \in A / M$ for each $k \in \mathbb{N}$. Consequently, $\Psi$ is an analytic $(A / M)$-valued function on $\mathbb{C} \cup\{\infty\}$. Since $A / M$ is a strongly galbed Hausdorff space by Proposition 2.1, $\Psi$ is a constant map, by Theorem 2.1 from [8].

To show that $\Psi(\lambda)=\theta_{A / M}$ for each $\lambda \in \mathbb{C}$, let $O$ be any neighbourhood of zero in $A$. Then there exist in $A$ a closed neighbourhood $O^{\prime}$ of zero and a balanced neighbourhood $V$ of zero such that $O^{\prime} \subset O$ and

$$
\left\{\sum_{k=0}^{n} \alpha_{k} v_{k}: v_{0}, \ldots, v_{n} \in V\right\} \subset O^{\prime}
$$

for each $n \in \mathbb{N}$. Moreover, there are $\mu_{z} \in \mathbb{C} \backslash\{0\}$ and $\rho_{V}>0$ such that

$$
\left(\frac{z}{\mu_{z}}\right)^{k} \in \rho_{V} V
$$

for each $k \in \mathbb{N}$. If now $|\lambda|>\max \left\{\frac{\left|\mu_{z}\right|}{\alpha}, \rho_{V}, \frac{\rho_{V}}{\alpha_{0}}\right\}$, then

$$
\left|\frac{\rho_{V}}{\lambda} \frac{\alpha^{k}}{\alpha_{k}}\left(\frac{\mu_{z}}{\alpha \lambda}\right)^{k}\right|<1
$$

for each $k \in \mathbb{N}$ and

$$
v_{k}(\lambda)=\frac{z^{k}}{\alpha_{k} \lambda^{k+1}}=\frac{1}{\rho_{V}} \frac{\rho_{V}}{\lambda} \frac{\alpha^{k}}{\alpha_{k}}\left(\frac{\mu_{z}}{\alpha \lambda}\right)^{k}\left(\frac{z}{\mu_{z}}\right)^{k} \in \frac{1}{\rho_{V}}\left[\frac{\rho_{V}}{\lambda} \frac{\alpha^{k}}{\alpha_{k}}\left(\frac{\mu_{z}}{\alpha \lambda}\right)^{k}\right] \rho_{V} V \subset V
$$

for each $k \in \mathbb{N}$, because $V$ is balanced. Therefore,

$$
\sum_{k=0}^{n} \frac{z^{k}}{\lambda^{k+1}}=\sum_{k=0}^{n} \alpha_{k} v_{k}(\lambda) \in O^{\prime}
$$

for each $n \in \mathbb{N}$. Since $O^{\prime}$ is closed, we have

$$
z(\lambda)^{-1}=\sum_{k=0}^{\infty} \frac{z^{k}}{\lambda^{k+1}}=\lim _{n \rightarrow \infty} \sum_{k=0}^{n} \alpha_{k} v_{k}(\lambda) \in O^{\prime} \subset O,
$$

whenever $|\lambda|>\max \left\{\frac{\left|\mu_{z}\right|}{\alpha}, \rho_{V}, \frac{\rho_{V}}{\alpha_{0}}, R\right\}$. Hence,

$$
\lim _{|\lambda| \rightarrow \infty} z(\lambda)^{-1}=\theta_{A}
$$

and

$$
\lim _{|\lambda| \rightarrow \infty} \Psi(\lambda)=\lim _{|\lambda| \rightarrow \infty} \pi_{M}\left(z(\lambda)^{-1}\right)=\pi_{M}\left(\lim _{|\lambda| \rightarrow \infty} z(\lambda)^{-1}\right)=\theta_{A / M} .
$$

Thus, $\Psi(\lambda)=\theta_{A / M}$ or $a(\lambda) \in M$ for each $\lambda \in \mathbb{C}$. Therefore,

$$
e_{A}=-\left(a(\lambda) z(\lambda)-e_{A}\right)+a(\lambda) z(\lambda) \in M,
$$


which is not possible. Consequently, for every $z \in Z(A)$ there is a $\lambda_{z} \in \mathbb{C}$ such that $z=\lambda_{z} e_{A}$. Hence, $Z(A)$ is isomorphic to $\mathbb{C}$.

To show that the isomorphism $\rho$ defined by $\rho(z)=\lambda_{z}$ for each $z \in Z(A)$ is continuous, let $O$ be a neighbourhood of zero in $\mathbb{C}$. Then there exists an $\epsilon>0$ such that

$$
O_{\epsilon}=\{\lambda \in \mathbb{C}:|\lambda|<\epsilon\} \subset O .
$$

Let $\lambda_{0} \in O_{\epsilon} \backslash\{0\}$. Since $A$ is a Hausdorff space, there exists a balanced neighbourhood $V$ of zero of $A$ such that $\lambda_{0} e_{A} \notin V$. But then also

$$
\lambda_{0} e_{A} \notin V^{\prime}=V \cap Z(A) .
$$

If $\left|\lambda_{z}\right| \geq\left|\lambda_{0}\right|$, then $\left|\lambda_{0} \lambda_{z}^{-1}\right| \leq 1$ and therefore, $\lambda_{0} e_{A}=\left(\lambda_{0} \lambda_{z}^{-1}\right) z \in V^{\prime}$ for each $z \in V^{\prime}$, which is not possible. Hence, $\lambda_{z} \in O$ for each $z \in V^{\prime}$. Thus, $\rho$ is continuous ( $\rho^{-1}$ is continuous because $Z(A)$ is a topological linear space in the subspace topology). Consequently, $A$ is central.

REMARK 3.2. Based on the previous Theorem 3.1 we can use the techniques of [3] to obtain the description of all closed maximal regular ideals of a unital $\sigma$-complete strongly galbed algebra $A$ in which all elements are bounded (see Theorem 3.6 in [3]). Similarly, by looking at the framework of Theorem 3.13 in [3], we can also show that such an algebra can be viewed as a subalgebra of the section algebra.

The author wishes to express his gratitude to Professor Anastasios Mallios for very useful conversations and terminological recommendations during the writing of the present article.

\section{References}

[1] Mart Abel, Description of closed maximal ideals in topological algebras, in: General Topological Algebras. Proc. of the Intern. Workshop held in Tartu, October 4-7, 1999. Est. Math. Soc., Tartu, 2001, 7-13.

[2] Mart Abel, Sectional Representations of Gelfand-Mazur Algebras, Sc. Math. Jap. (2001), 797-804.

[3] Mart Abel, Structure of Gelfand-Mazur algebras, Dissertationes Mathematicae Universitatis Tartuensis 31, Tartu University Press, Tartu, 2003.

[4] Mart Abel and Mati Abel, The center of topologically primitive exponentially galbed algebras, submitted.

[5] Mati Abel, On the Gelfand-Mazur theorem for exponentially galbed algebras, Tartu Ülik. Toimetised 899 (1990), 65-70.

[6] Mati Abel, Gelfand-Mazur algebras, in: Topological Vector Spaces, Algebras and Related Areas. Pitman Research Notes in Math. Series 316, Longman, Harlow, 1994, 116-129.

[7] Mati Abel, The center of primitive locally psedoconvex algebras, Bull. Belg. Math. Soc. Simon Stevin 11 (2004), 191-199.

[8] Mati Abel, Galbed Gelfand-Mazur algebras, Contemporary Mathematics, to appear.

[9] V. K. Balachandran, Topological Algebras, North-Holland Math. Studies 185, Elsevier, Amsterdam, 2000.

[10] F. F. Bonsall and J. Duncan, Complete Normed Algebras, Springer-Verlag, Berlin 1973. 
[11] H. G. Dales, Banach Algebras and Automatic Continuity, London Math. Soc. Monographs. New Series 24, Oxford Univ. Press, New York, 2000.

[12] I. N. Herstein, Noncommutative Rings, Wiley, New York, 1968.

[13] N. Jacobson, Structure of Rings, Amer. Math. Soc., Colloquium Publ. 37, Providence, Rhode Island, 1968 (revised edition).

[14] Th. W. Palmer, Banach Algebras and the General Theory of *-Algebras, Vol. I. Algebras and Banach algebras, Encyclopedia Math. Appl. 49, Cambridge Univ. Press, Cambridge, 1994.

[15] C. E. Rickart, General Topology of Banach Algebras, D. van Nostrand, Princeton, 1960.

[16] Y. Tsertos, On primitive topological algebras, Bull. Math. Greek Math. Soc. 28 (1987), 81-92.

[17] Y. Tsertos, On primitive A-convex algebras, Comment. Math. Prace Mat. 41 (2001), 203-219.

[18] Ph. Turpin, Espaces et opérateurs exponentiellement galbés, in: Séminaire Lelong (Analyse) Année 1973/74, Lect. Notes in Math. 474, Springer-Verlag, Berlin, 1975, 48-62.

[19] Ph. Turpin, Convexités dans les espaces vectoriels topologiques généraux, Dissertationes Math. (Rozprawy Mat.) 131 (1976).

[20] L. Waelbroeck, Topological Vector Spaces and Algebras, Lecture Notes in Math. 230, Springer-Verlag, Berlin, 1973. 